\title{
Influencia del aumento de la grasa dietaria sobre los lípidos séricos y el consumo de heno y extracto etéreo en novillos
}

\author{
Koza, G.A.'; Mussart, N.B. ${ }^{1}$; Rocchinotti, D. ${ }^{2}$; Gimenez, L. ${ }^{3}$; Coppo, J.A. ${ }^{1}$ \\ ${ }^{1}$ Cátedra de Fisiología, Facultad de Ciencias Veterinarias, UNNE, Sargento Cabral 2139, Corrientes (3400), \\ Argentina. Tel/fax 03783-425753. ${ }^{2}$ INTA Mercedes. ${ }^{3}$ INTA Corrientes. E-mail: fisiologia@vet.unne.edu.ar.
}

\begin{abstract}
Resumen
Koza, G.A.; Mussart, N.B.; Rocchinotti, D.; Gimenez, L.; Coppo, J.A.: Influencia del aumento de la grasa dietaria sobre los lípidos séricos y el consumo de heno y extracto etéreo en novillos. Rev. vet. 20: 2, 110-115, 2009. El objetivo fue verificar cambios provocados por la suplementación con semilla entera de algodón (SA, 18,9\% de lípidos), sobre el consumo de heno y extracto etéreo (EE), el EE en materia fecal y el perfil lipídico sérico en novillos. Se empleó un diseño cuadrado latino. Cuatro novillos mantenidos con heno fueron suplementados diariamente con diferentes niveles de SA: tratamientos: bajo $(0,6 \mathrm{~kg})$, medio $(1,2 \mathrm{~kg})$, alto $(1,8 \mathrm{~kg})$ y cero $(\sin \mathrm{SA})$. La experiencia abarcó 84 días. Muestras de materia fecal se colectaron a las $0,2,4,8,12,15$ y 18 horas post-ingesta. La sangre se extrajo el día 21 y con el suero obtenido se determinaron colesterol total (CT), triglicéridos (TG), colesterol ligado a lipoproteínas de alta y baja densidad (C-HDL, C-LDL), lipoproteínas alfa (LA) y beta (LB), así como la relación beta/alfa (RBA). Los resultados se procesaron para obtener estadísticas descriptivas, contrastes ortogonales y comparaciones de medias (Duncan). El aumento progresivo de SA dietaria provocó disminuciones (no significativas) del consumo de heno, que fue elevado en el tratamiento cero $(5,028 \mathrm{~kg})$ con relación a bajo $(4,800 \mathrm{~kg})$, medio $(4,629 \mathrm{~kg})$ y alto $(3,614 \mathrm{~kg})$. El consumo de $\mathrm{EE}$ aumentó con la suplementación $(\mathrm{p}=0,043)$. El nivel de aparición de EE en heces fue mayor $(\mathrm{p}=0,00014)$ en alto, seguido de medio, bajo y cero. CT se elevó $(\mathrm{p}=0,001)$ desde cero $(1,26 \mathrm{~g} / \mathrm{l})$ hacia bajo $(1,86 \mathrm{~g} / \mathrm{l})$, medio $(2,07 \mathrm{~g} / \mathrm{l}) \mathrm{y}$ alto $(2,52 \mathrm{~g} / \mathrm{l})$; similar evolución registraron $\mathrm{TG}(0,34 ; 0,36 ; 0,42$ y $0,69 \mathrm{~g} / 1$ respectivamente). También aumentaron progresivamente C-HDL ( $\mathrm{p}=0,001), \mathrm{C}-\mathrm{LDL}(\mathrm{p}=0,004)$ y LA $(\mathrm{p}=0,05)$. LB y RBA declinaron desde alto a cero $(\mathrm{p}=0,046$ y $\mathrm{p}=0,076)$. Se concluye que la incorporación de SA a la ración de novillos genera disminución del consumo de heno, aumento del consumo de EE e incremento de EE en heces, así como elevaciones de CT, TG, C-HDL, CLDL y LA, con disminución de LB y RBA.
\end{abstract}

Palabras clave: novillo, suplementación, semilla de algodón, consumo de heno, lípidos séricos.

\begin{abstract}
Koza, G.A.; Mussart, N.B.; Rocchinotti, D.; Gimenez, L.; Coppo, J.A.: Influence of dietary fat increase on serum lipids and the consumption of hay and ethereal extract in steers. Rev. vet. 20: 2, 110-115, 2009. The objective was to verify changes caused by the supplementation with whole cottonseed (WC, 18.9\% lipids), on the hay and ethereal extract (EE) consumption, as well as on the fecal level of EE and the serum lipid profile in steers. A latin square design was used. Four steers fed on hay were daily supplemented with different levels of WC: treatments low $(0.6 \mathrm{~kg})$, medium $(1.2 \mathrm{~kg})$, high $(1.8 \mathrm{~kg})$ and zero (without WC). Experience lasted 84 days. Feces samples were collected at $0,2,4,8,12,15$ and 18 hours after food intake. Blood was obtained at day 21, and serum was used to determine total cholesterol (TC), triglycerides (TG), cholesterol bound to high and low density lipoproteins (C-HDL, C-LDL, respectively), alpha and beta lipoproteins (AL, BL), as well as the beta/alpha ratio (BAR). Results were processed to obtain descriptive statistics, orthogonal contrasts, and mean comparisons (Duncan). The progressive increase of dietary WC caused decreases (not significant) of hay consumption, which was increased in the treatment zero $(5.028 \mathrm{~kg}$ ) compared to low $(4.800 \mathrm{~kg})$, medium $(4.629 \mathrm{~kg})$ and high $(3.614 \mathrm{~kg})$. EE consumption increased with supplementation $(\mathrm{p}=0.043)$. The level of $E E$ in feces increased $(\mathrm{p}=0.00014)$ on high, followed by medium, low, and zero. TC increased $(\mathrm{p}=0.001)$ from zero $(1.26 \mathrm{~g} / \mathrm{l})$ to low $(1.86 \mathrm{~g} / 1)$, medium $(2.07 \mathrm{~g} / \mathrm{l})$ and high $(2.52 \mathrm{~g} / \mathrm{l})$; similar evolution was registered by TG $(0.34$;
\end{abstract}

Recibido: 30 octubre 2009 / Aceptado: 23 noviembre 2009 
0.36; 0.42 and $0.69 \mathrm{~g} / 1$, respectively). C-HDL, $\mathrm{C}-\mathrm{LDL}$, and $\mathrm{AL}$ also increased progressively $(\mathrm{p}=0.001 ; \mathrm{p}=0.004 ; \mathrm{p}=0.05)$. BL and BAR declined from high to zero $(\mathrm{p}=0.046$ and $\mathrm{p}=0.076)$. It can be concluded that incorporation of $\mathrm{WC}$ to the ration of steers causes hay consumption decrease, EE consumption increase, and fecal EE increment, as well as TC, TG, C-HDL, C$\mathrm{LDL}$ and $\mathrm{AL}$ increase, and BL and BAR decrease.

Key words: steer, supplementation, whole cottonseed, hay consumption, serum lipid.

\section{INTRODUCCIÓN}

En los forrajes que integran las raciones consumidas habitualmente por los rumiantes, las grasas existen en cantidades relativamente pequeñas ${ }^{10}$. La adición de lípidos a la dieta se realiza por medio de semillas oleaginosas o de manera directa, añadiendo grasas o aceites de origen animal o vegetal ${ }^{30}$.

La semilla entera de algodón (SA) es un importante recurso alimenticio, de buen valor nutritivo y bajo costo en el nordeste argentino. Contiene de 18 a $24 \%$ de extracto etéreo (EE), ostentando un buen balance entre proteína bruta (PB), de 18 a $24 \%$ de la materia seca (MS) y energía metabolizable (3,5 Mcal/kg de MS) ${ }^{26}$ . La semilla entera con cubierta es la más usada como alimento animal, aportando fibra, proteínas y energía; la fibra se halla en la cubierta ( $10 \%$ del peso de la semilla entera) y posee elevado porcentaje de celulosa y alta digestibilidad. Las proteínas se hallan en el core y la energía en el aceite que posee la semilla ${ }^{7}$.

En los rumiantes, las grasas aportadas por la ración permiten reducir los niveles de carbohidratos, estimular la utilización de la fibra y, mediante la biohidrogenación, mejorar la recuperación de energía ${ }^{14,25}$. La suplementación con grasas puede ejercer influencias negativas sobre la función ruminal, pero limitando su ingesta (al 5\% de la ración), los efectos se minimizan.

La grasa aportada no debe reducir la digestión de la fibra vegetal, tarea realizada por los microorganismos ruminales ${ }^{29}$. Tampoco el agregado de lípidos a la ración debería alterar la digestión ni la disponibilidad de los otros nutrientes ${ }^{25}$. Cuando el rumiante ingiere grandes cantidades de aceites poliinsaturados, éstos pueden superar los límites de la hidrólisis y de la hidrogenación, escapando al rumen sin sufrir modificación alguna ${ }^{3}$.

El objetivo del presente trabajo fue verificar los cambios provocados por la SA a nivel de consumo de heno, consumo de la fracción EE, nivel de la fracción EE en la materia fecal y perfil lipídico sanguíneo.

\section{MATERIAL Y MÉTODOS}

Bajo un diseño cuadrado latino balanceado, cuatro novillos cruza cebú fueron alimentados con heno de Cynodon nlemfuensis y suplementados diariamente con diferentes niveles de SA, los cuales constituyeron respectivamente los tratamientos bajo $(0,6 \mathrm{~kg})$, medio
$(1,2 \mathrm{~kg})$, alto $(1,8 \mathrm{~kg})$ y cero $(\sin \mathrm{SA})$. En la Tabla $1 \mathrm{se}$ consigna al análisis químico practicado al suplemento $y$ al heno empleados en la experiencia.

El heno fue ofertado ad libitum, en cantidad suficiente para permitir un rechazo superior al $10 \%$. La duración del ensayo fue de 84 días (4 períodos de 21 días cada uno). El concentrado y el heno se administraron a los animales en una toma diaria, por la mañana (7 AM). El día 1 de cada período se procedió al registro de peso de cada animal, en una balanza digital móvil individual.

Las muestras de heno y suplemento para la valoración del consumo, se analizaron según la técnica de Moore modificada ${ }^{23}$. Las muestras de materia fecal (200 g aproximadamente) se colectaron a las 0, 2, 4, 8, 12,15 y 18 horas posteriores a la ingesta del suplemento más el heno, entre los días 20 y 21 del ensayo. El EE del heno, suplemento y materia fecal se midió por el método proximal de Weende.

Las muestras de sangre se obtuvieron el día 21 de cada período, por venopunción yugular. Con el suero se realizaron las pruebas bioquímicas relacionadas al perfil lipídico: colesterol total (CT), triglicéridos (TG), colesterol ligado a lipoproteínas de baja y alta densidad (C-LDL y C-HDL), según técnicas de laboratorio convencionales. También se evaluaron las lipoproteínas alfa y beta (LA y LB), así como la relación beta-alfa (RBA), por electroforesis en gel de agarosa, coloración Fat-Red 7B y cuantificación por densitometría.

Con el auxilio del programa SAS Versión VIII ${ }^{27}$, se obtuvieron estadísticas descriptivas, contrastes ortogonales (efectos lineales, cuadráticos y cúbicos) y comparaciones de medias (test de Duncan). El nivel de significancia de la probabilidad alfa fue fijado en 0,05 .

\section{RESULTADOS Y DISCUSIÓN}

El peso vivo medio de los animales bajo ensayo no registró diferencias estadísticas significativas atribui- 
Tabla 2. Consumo de heno, de suplemento y de materia seca total en cada tratamiento. Consumo de la Fracción EE.

\begin{tabular}{|c|c|c|c|c|c|c|c|c|}
\hline tratamiento & cero & bajo & medio & alto & $\mathrm{P}$ & $\mathrm{E}$ & $\mathrm{CV}$ & $\mathrm{DE}$ \\
\hline PVM (kg) & 365,125 & 362,125 & 365,250 & 355,625 & - & - & - & - \\
\hline \multicolumn{9}{|l|}{ consumo de heno } \\
\hline en $\mathrm{kg}$ de MS & 5,028 & 4,800 & 4,629 & 3,614 & 0,195 & - & 18,866 & 0,875 \\
\hline de EE /día, kg de MS & 0,084 & 0,080 & 0,076 & 0,060 & 0,369 & - & 19,763 & 0,597 \\
\hline de EE/ día, \% PV & 0,023 & 0,022 & 0,021 & 0,017 & 0,289 & - & 10,563 & 0,143 \\
\hline \multicolumn{9}{|l|}{$\begin{array}{l}\text { consumo de } \\
\text { suplemento (SA) }\end{array}$} \\
\hline en $\mathrm{kg}$ de MS & 0 & 0,558 & 1,117 & 1,675 & - & - & 0,089 & 0,008 \\
\hline de EE/día, kg de MS & 0 & 0,106 & 0,212 & 0,317 & - & - & 6,479 & 0,023 \\
\hline de EE/ día, \% PV & 0 & 0,029 & 0,058 & 0,089 & - & - & 9,569 & 0,014 \\
\hline \multicolumn{9}{|l|}{$\begin{array}{l}\text { consumo total } \\
\text { heno }+ \text { SA }\end{array}$} \\
\hline en $\mathrm{kg}$ MS & 5,028 & 5,356 & 5,856 & 5,289 & 0,705 & - & 15,909 & 0,852 \\
\hline de EE/día, kg de MS & $0,084^{\mathrm{A}}$ & $0,185^{\mathrm{B}}$ & $0,288^{\mathrm{C}}$ & $0,377^{\mathrm{D}}$ & 0,043 & $\mathrm{~L}, \mathrm{C}, \mathrm{Q}$ & 13,568 & 0,529 \\
\hline de EE/ día, \% PV & $0,023^{\mathrm{A}}$ & $0,051^{\mathrm{A}}$ & $0,079^{\mathrm{B}}$ & $0,106^{\mathrm{C}}$ & 0,039 & $\mathrm{~L}, \mathrm{C}, \mathrm{Q}$ & 12,361 & 0,129 \\
\hline de $\mathrm{EE} \%$ de la dieta & $1,650^{\mathrm{A}}$ & $3,452^{\mathrm{B}}$ & $4,917^{\mathrm{C}}$ & $7,126^{\mathrm{D}}$ & 0,05 & $\mathrm{~L}, \mathrm{Q}$ & 6,521 & 0,597 \\
\hline
\end{tabular}

P: probabilidad alfa $(<0,05)$; E: efecto; CV: coeficiente de variación; DE: desvío estándar; PVM: peso vivo medio; MS: materia seca; EE: extracto etéreo; PV: peso vivo; L: lineal, C: cuadrático, Q: cúbico. Letras distintas expresan diferencias significativas (test de Duncan).

bles a los efectos período ni animal $(\mathrm{p}=0,507)$, lo cual revela la homogeneidad del peso de los sujetos experimentales a lo largo de la experiencia, que fue de 362,03 $\mathrm{kg}$. El efecto tratamiento (suplementación con SA) tampoco influyó significativamente sobre esta variable.

Los efectos período y animal no afectaron de manera significativa ninguna de las variables relacionadas al consumo. Se registraron disminuciones (no significativas, $p=0,195$ ) del consumo de heno ante la creciente incorporación de SA en la ración. Los mayores niveles de consumo se observaron en el tratamiento cero $(5,028$ $\mathrm{kg})$ con relación a los tratamientos bajo $(4,800 \mathrm{~kg})$, medio $(4,629 \mathrm{~kg})$ y alto $(3,614 \mathrm{~kg})$. La Figura 1 muestra las modificaciones del consumo de heno, de suplemento y de MS total de acuerdo al tratamiento. En la Tabla 2 se registran los resultados del consumo de heno, consumo de suplemento y consumo total, en base a MS y en porcentaje al peso vivo.

Algunos autores registraron incrementos del consumo de heno en $\%$ del peso vivo (PV), del consumo total de MS y del consumo total de MS en \% PV al emplear SA como suplemento, en animales intactos en confinamiento ${ }^{19}$, sin verificar variaciones del consumo de heno en base a la MS.

Otros observaron que los consumos de MS y MO no fueron modificados por la incorporación de aceite de lino a la ración de bovinos de carne con niveles variables de la relación forraje-concentrado en la dieta ${ }^{28}$ . Estos resultados fueron confirmados en trabajos realizados en vacas lecheras ${ }^{12}$ y cabras ${ }^{9}$.

La SA utilizada en la presente experiencia contenía un porcentaje $18,9 \%$ de EE. El consumo total de EE se vio modificado significativamente $(p=0,043)$ por la suplementación con SA; el tratamiento alto registró los mayores niveles de consumo de esta fracción, disminuyendo hacia el tratamiento cero. El aumento del consumo de suplemento, por elevación de los niveles diarios de SA en la ración, incorporó mayores porcentajes de grasas a la dieta. Los niveles crecientes de SA $(0,559$ a $1,679 \mathrm{~kg}$ de SA en base seca), adicionaron al rumen de 1,94 a $5,80 \%$ de grasas (del tratamiento bajo al alto). La Figura 2 revela la evolución del consumo de la fracción EE del heno, suplemento y consumo total.

En otras investigaciones se postuló que las modificaciones en el consumo de heno se deberían, por un lado, al efecto de sustitución que ejerce el suplemento (SA) ocasionando la reducción en la ingesta de heno, y por el otro, al elevado contenido de grasa que aporta la $\mathrm{SA}^{22}$, sugiriendo que en dietas con altas proporciones de forraje, el consumo y digestibilidad de los nutrientes

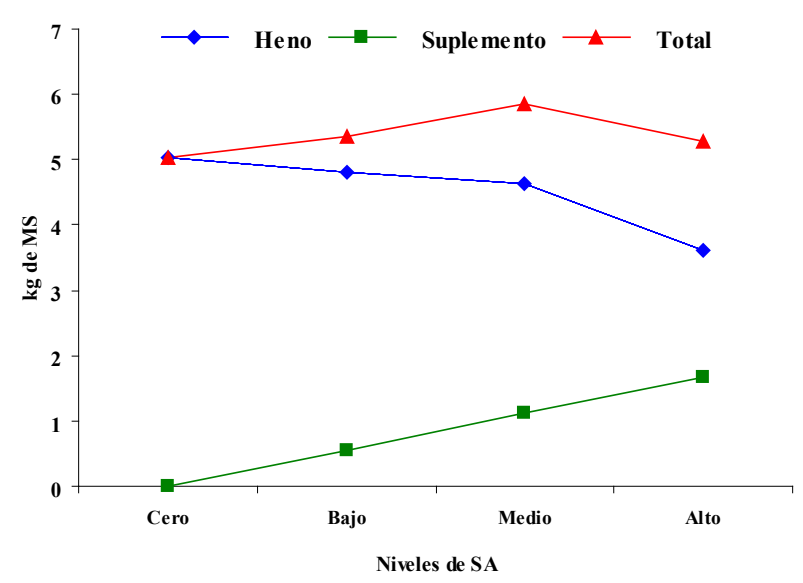

Figura 1. Consumo de heno, de suplemento y de materia seca total. 
se deprimen con la incorporación de más del $5 \%$ de grasas ${ }^{24}$, que ocasionaría la disminución de la actividad y el número de microorganismos celulolíticos ${ }^{11}$.

El nivel de aparición del EE en materia fecal fue significativamente mayor $(\mathrm{p}=0,00014)$ en el tratamiento alto $(1,77 \%)$, seguido por medio, bajo y cero $(1,45$; 1,13 y $0,96 \%$ respectivamente), variaciones que se esquematizan en la Figura 3.

La SA no escapa a la degradación ruminal ${ }^{8}$. Se afirma que la degradación ruminal de la SA se ve favorecida por la regurgitación y remasticación (rumia), más aún cuando se administra con dietas de elevado contenido en fibra ${ }^{8,15,21}$. Esto explicaría el bajo porcentaje de SA entera hallado a nivel intestinal ${ }^{7}$, en disidencia con autores que sugieren que la SA escapa a la hidrogenación ruminal debido a su cubierta natural ${ }^{1}$.

En el presente ensayo, la incorporación de SA a la ración generó aumentos significativos de los parámetros séricos relacionados al metabolismo lipídico. El $\mathrm{CT}$ se elevó significativamente $(\mathrm{p}=0,001)$ desde el tratamiento cero $(1,26 \mathrm{~g} / \mathrm{l})$ hacia bajo $(1,86 \mathrm{~g} /)$, medio $(2,07$ $\mathrm{g} / \mathrm{l})$ y alto $(2,52 \mathrm{~g} / \mathrm{l})$. Elevaciones lineales del colesterol sérico fueron observadas en bovinos que recibieron niveles crecientes de SA (de 1,50 a 2,25 kg), asociadas a niveles decrecientes de sorgo molido ${ }^{16,17}$.

TG, C-HDL y C-LDL ostentaron incrementos lineales al aumentar el nivel de incorporación de SA a la ración. En cabras que recibieron dietas con elevado tenor graso en base a aceites de palma y oliva en baja y alta proporción, los animales que recibieron esta última, registraron elevaciones significativas de $\mathrm{CT}$ y de $\mathrm{C}-\mathrm{HDL}^{2}$.

Los niveles plasmáticos de TG, CT, C-HDL y CLDL se incrementaron significativamente en vaquillas de reposición que recibieron suplementación invernal tanto en base a SA como a soja entera $(p=0,0001)$, en relación a un lote control, tanto en el primero como segundo inviernos ${ }^{18}$. Cifras más bajas de TG fueron

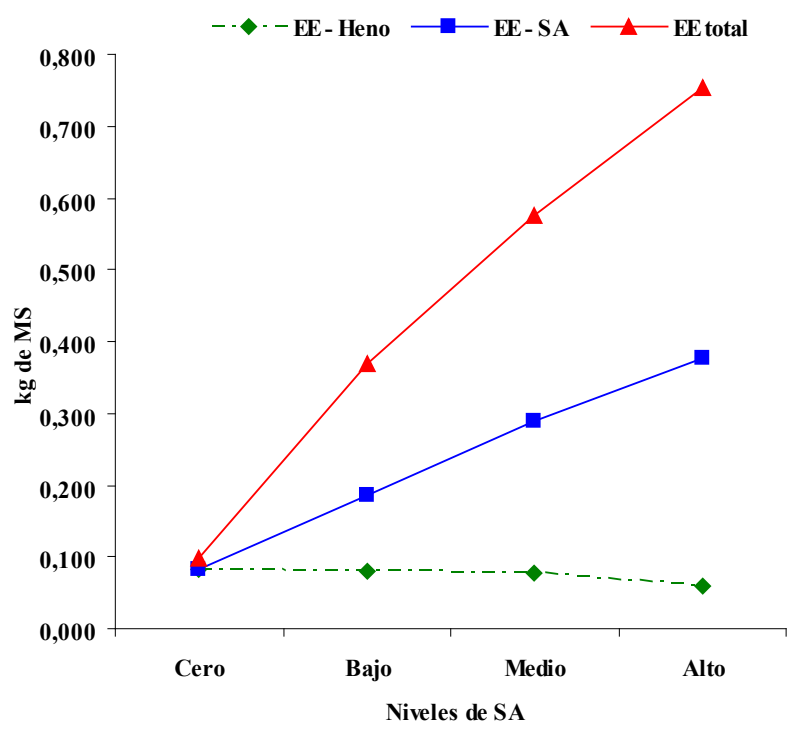

Figura 2. Consumo de la fracción extracto etéreo. registradas en ensayos de suplementación energéticoproteica realizadas en el nordeste argentino ${ }^{5}$.

Cantidades crecientes de SA en la dieta de novillos produjeron elevaciones progresivas de TG séricos $(0,34 ; 0,36 ; 0,42$ y $0,69 \mathrm{~g} / \mathrm{l})$. A la inversa, los niveles séricos de TG disminuyeron al reducirse el aporte lipídico de la dieta en becerras ${ }^{20}$.

Aumentos de C-HDL y C-LDL fueron registrados en novillos suplementados con SA y sorgo molido ${ }^{16,17}$. En concordancia, la administración de ácidos grasos en forma de jabones cálcicos redundó en incrementos séricos de HDL, VLDL, CT y TG ${ }^{13}$. El predominio de C-HDL sobre C-LDL depende del patrón lipoproteico de la especie ${ }^{6}$.

El comportamiento de las fracciones lipoproteicas aquí registrado, fue similar al observado en un ensayo donde vaquillas de recría fueron separadas en lotes que recibieron una ración en base a SA, soja entera (SS) y un control solo a pasto $(\mathrm{CN})$ respectivamente; la tasa plasmática promedio de LA fue significativamente influenciada $(\mathrm{p}=0,028)$ por la suplementación, tanto con SA $(85,97 \%)$ como con SS $(85,73 \%)$ en comparación al lote CN (84,13\%). Inversamente, LB y RBA fueron mayores en $\mathrm{CN}(15,81$ y $0,19 \%$ respectivamente) que en SA $(13,89$ y $0,16 \%$ respectivamente) y SS $(14,27$ y $0,17 \%$ respectivamente) ${ }^{18}$. Es generalizadamente admitido que en bovinos los niveles séricos de LA son fisiológicamente mayores que los de $\mathrm{LB}^{6,20}$.

En la Tabla 3 se consignan los niveles séricos de los parámetros bioquímicos incluidos dentro del perfil lipídico analizado en la experiencia. En otros ensayos de suplementación de distintas categorías bovinas con SA, se hallaron descensos de los niveles de LB y RBA, así como aumentos de LA, TG y CT ${ }^{4,16}$.

En conclusión, la incorporación de diferentes niveles de SA a la ración de novillos generó disminución del consumo de heno, incremento del consumo de la fracción EE y aumento de la aparición de EE en la materia fecal, acompañados de elevaciones séricas de CT, TG, C-HDL, C-LDL y LA, así como disminuciones de LB y RBA.

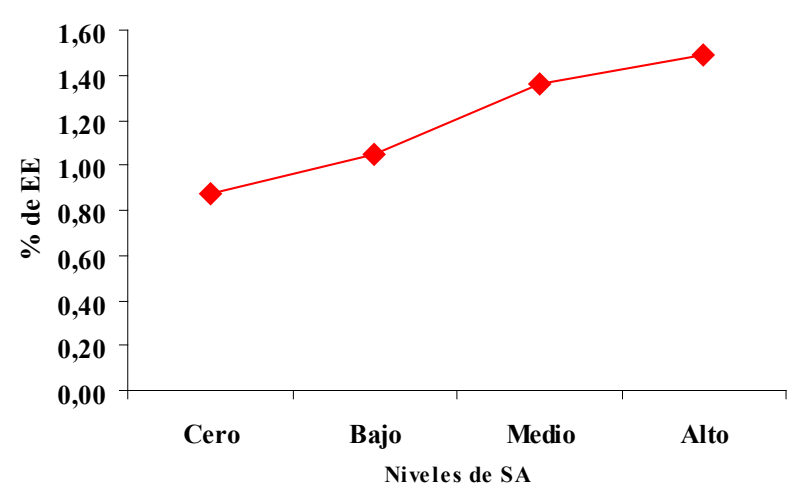

Figura 3. Nivel de aparición de EE en materia fecal. 
Tabla 3. Variables relacionadas al metabolismo lipídico.

\begin{tabular}{|c|c|c|c|c|c|c|c|c|c|}
\hline \multirow{2}{*}{ variable } & \multicolumn{4}{|c|}{ tratamientos } & \multirow{2}{*}{$\mathrm{P}$} & \multirow{2}{*}{$\mathrm{E}$} & \multirow{2}{*}{$\mathrm{CV}$} & \multirow{2}{*}{$\mathrm{EE}$} & \multirow{2}{*}{$\mathrm{DE}$} \\
\hline & cero & bajo & medio & alto & & & & & \\
\hline$\overline{\mathrm{C}-\mathrm{HDL}(\mathrm{g} / \mathrm{l})}$ & $0,80^{\mathrm{A}}$ & $1,34^{\mathrm{B}}$ & $1,70^{\mathrm{C}}$ & $1,91^{\mathrm{C}}$ & 0,001 & $\mathrm{~L}, \mathrm{C}, \mathrm{Q}$ & 14,24 & 0,10 & 0,20 \\
\hline $\mathrm{C}-\mathrm{LDL}(\mathrm{g} / \mathrm{l})$ & $0,10^{\mathrm{A}}$ & $0,14^{\mathrm{A}}$ & $0,27^{\mathrm{B}}$ & $0,47^{\mathrm{C}}$ & 0,004 & $\mathrm{C}, \mathrm{Q}$ & 30,65 & 0,03 & 0,22 \\
\hline $\mathrm{CT}(\mathrm{g} / \mathrm{l})$ & $1,26^{\mathrm{A}}$ & $1,86^{\mathrm{B}}$ & $2,07^{\mathrm{B}}$ & $2,52^{\mathrm{C}}$ & 0,003 & $\mathrm{~L}, \mathrm{C}, \mathrm{Q}$ & 13,13 & 0,12 & 0,25 \\
\hline TG (g/l) & $0,33^{\mathrm{A}}$ & $0,53^{\mathrm{B}}$ & $0,64^{\mathrm{B}}$ & $0,83^{\mathrm{C}}$ & 0,003 & $\mathrm{C}, \mathrm{Q}$ & 18,07 & 0,05 & 0,10 \\
\hline LA $(\%)$ & $83,85^{\mathrm{A}}$ & $89,25^{\mathrm{AB}}$ & $90,77^{\mathrm{B}}$ & $93,27^{\mathrm{B}}$ & 0,05 & $\mathrm{C}$ & 4,23 & 1,89 & 3,77 \\
\hline LB (\%) & $16,15^{\mathrm{A}}$ & $10,85^{\mathrm{AB}}$ & $9,25^{\mathrm{B}}$ & $6,75^{\mathrm{B}}$ & 0,04 & $\mathrm{C}$ & 29,14 & 1,52 & 3,05 \\
\hline RBA (score) & $0,17^{\mathrm{A}}$ & $0,11^{\mathrm{AB}}$ & $0,09^{\mathrm{B}}$ & $0,09^{\mathrm{B}}$ & 0,07 & $\mathrm{C}$ & 33,65 & 0,02 & 0,04 \\
\hline
\end{tabular}

P: probabilidad alfa $(<0,05)$; E: efecto; CV: coeficiente de variación; EE: error estándar; DE: desvío estándar; L: lineal, C: cuadrático, Q: cúbico. C-HDL y C-LDL: colesterol unido a lipoproteínas de alta y baja densidad; CT: colesterol total; TG: triglicéridos; LA y LB: lipoproteínas alfa y beta; RBA: relación beta/alfa. Letras distintas expresan diferencias significativas (test de Duncan).

Agradecimientos. Al Ing.Agr. G.Tortarolo, al PhD. M.V. O.Balbuena y al personal del INTA-Colonia Benítez (Chaco) por permitir y colaborar en el desarrollo del presente trabajo. A la Bioq. G. Somma de Feré y al personal del laboratorio del INTA-Mercedes (Corrientes), por el procesamiento de las muestras de alimentos y materia fecal. Al M.V. M. Navamuel por su asistencia en el procesamiento estadístico de los datos.

\section{REFERENCIAS}

1. Baldwin RL, Allison ML. 1983. Rumen metabolism. $J$ Anim Sci 57(Suppl. 2): 461.

2. Beynen AC, Schonewille JT, Terpstra AM. 2000. Influence of amount and type of dietary fat on plasma cholesterol concentration in goats. Small Ruminant Res 35: 141-147.

3. Byers FM, Schelling GT. 1988. Los lípidos en la nutrición de los rumiantes. En: El rumiante. Fisiología digestiva y nutrición (Church DC, Ed.), Acribia, Zaragoza, p. 339355.

4. Coppo JA, Maccio OA, Scorza SH, Mussart NB. 1993. Desórdenes metabólicos en bovinos suplementados con semilla entera de algodón. Arch Zoot 42: 301-303.

5. Coppo JA, Scorza SH, Mussart NB. 1995. Biochemical profiles of argentine cattle supplemented with cottonseed. RIA 25: 91-102.

6. Coppo JA. 2008. Fisiología comparada del medio interno, $2^{\circ}$ ed., EUCASA, Salta (Argentina), 310 p.

7. Coppock CE, West J, Moya JR, Nave DH, Labore JM, Thompson KG, Rowe LD, Gates CE. 1985. Effects of amount of whole cottonseed on intake, digestibility, and physiological responses of dairy cows. J Dairy Sci 69: 2248-2258.

8. Coppock CE, Lanham JK, Horner JI. 1987. A review of the nutritive value and utilization of whole cottonseed, cottonseed meal and associated by-products by dairy cattle. Anim Feed Sci Techn 18: 89-93.

9. Chilliard Y, Chabosseau JM, Rouel J, Capitan P, Gominard C, Gaborit P, Juaneda P, Ferlay A. 2002. Interactions between forage nature and sunflower or linseed oil supplementation on goat milk fatty acids of interest for human nutrition. Gassland Sci Eur 7: 548-549.
10. Church DC. 1988. El rumiante. Fisiología digestiva y nutrición, Acribia, Zaragoza, $641 \mathrm{p}$.

11. Devendra C, Lewis D. 1974. The interaction between dietary lipids and fibre in the sheep. 2. Digestibility studies. Anim Prod 19: 67-76.

12. Dhiman TR, Satter LD, Pariza T, Galli MW, Albright K, Tolosa X. 2000. Conjugated linoleic acid (CLA) content of milk form cows offered diets rich in linoleic and linolenic acid. J Dairy Sci 83: 1016-1027.

13. Espinoza JL, Ramirez-Godinez JA, Simental SS, Jiménez J, Ramirez R, Palacios A, Delun R. 1997. Effects of calcium soaps of fatty acids on serum hormones and lipid metabolites in pelibuey ewes. Small Ruminant Res 26: 61-68.

14. Gagliostro GA, García SC, Santini FJ, Dillon A, Lavandera SE. 1995. Suplementación con concentrados protei$\cos$ de distinta degradabilidad ruminal en vacas lecheras en pastoreo. Rev Arg Prod Anim 15: 517-520.

15. Huerta-Leidenz NO, Cross HR, Lunt DK, Pelton LS, Savell JW, Smith SB. 1991. Growth, carcass traits, and fatty acid profiles of adipose tissues from steers fed cottonseed. J Anim Sci 69: 3665-3672.

16. Koza GA, Balbuena O, Kucseva CD, Coppo NB, Coppo JA. 2000. Efectos de la suplementación con semilla de algodón sobre los lípidos plasmáticos y el $\mathrm{pH}$ ruminal de novillos cruza cebú. Anales $V$ Reunión Latinoamericana de Fisiología Veterinaria, Buenos Aires, p. 15-17.

17. Koza GA, Balbuena O, Kucseva CD, Mussart NB, Coppo JA. 2001. Cambios del lipidograma en novillos suplementados con semilla de algodón. Anales Reunión de Comunicaciones Cientificas y Técnicas UNNE, Corrientes (Argentina), comunicación 021. On line: http://www.unne. edu.ar/cyt/2001/cyt.htm.

18. Koza GA, Mussart NB, Fioranelli SA, Alvarez GM, Coppo JA. 2009. Respuesta de indicadores nutricionales en vaquillas suplementadas con semillas de soja y algodón en la Provincia del Chaco, Argentina. Rev Vet 20: 15-21.

19. Kucseva CD, Balbuena O, Slanac AL, Schreiner J, Somma GR, Koza GA, Rochinotti D. 2001. Efectos de niveles de semilla de algodón en el suplemento sobre el consumo de heno de novillitos. Rev Arg Prod Anim 21 (Supl. 1): 5-6. 
20. López-Ortega AA, Márquez YC, Mendoza CA, Ferraro SM, Márquez AA. 2008. Perfil lipídico en becerras mestizas carora durante el primer año de vida, en época de lluvias y de sequía, en Venezuela. Rev Vet 19: 2-7.

21. Maman A, Alio A, Hale WH, Swingle SR. 1989. Integrity of whole cottonseed in the digestive tract of steers. Ariz Agric Exp Tech 47: 433-434.

22. McLennan SR, Plasto AW, Doogran VJ, Dillon RD. 1998. Whole cottonseed and cottonseed meal supplements for cattle given a hay based diet. Anim Produc Australia 22: 111-114.

23. Moore JE. 1981. Procedure for determining voluntary intake and nutrient digestibility of hay with sheep, Ed Dep Anim Sci Univ Florida, Gaynesville (USA), 27 p.

24. Moore JE, Swingle RS, Hale WH. 1986. Effects of whole cottonseed, cottonseed oil and animal fat on digestibility of wheat straw diets by steers. J Anim Sci 63: 1267-1273.

25. Palmquist DL. 1996. Utilización de lípidos en dietas de rumiantes. On line: www.etsia.upm.es/Fedna/ capitulos/96capituloIII.pdf.
26. Poore MH, Rogers G. 1995. Feeding whole cottonseed and other cotton by-products to beef cattle. Vet Med 90 : 1077-1087.

27. SAS Institute Inc. 1987. SAS/STAT guide for personal computers, Version 8. SAS Institute INC. Cary, NC (USA), $72 \mathrm{p}$.

28. Ueda K, Ferlay A, Chabrot J, Loor JJ, Chilliard Y, Doreau M. 2003. Effect of linseed oil supplementation on ruminal digestion in dairy cows fed diets with different forage: concentrate rations. J Dairy Sci 86: 3999-4007.

29. Vargas GA, Kolver ES. 1997. Microbial and animal limitations to fiber digestion and utilization. $J$ Nutr 127 (suppl 5): 819-823.

30. Zinn RA, Plascencia A. 1993. Interaction of whole cottonseed and supplemental fat on digestive function in cattle. $J$ Anim Sci 71: 11-17.

\section{Revista Veterinaria obtuvo el máximo nivel de categorización del CAICYT-CONICET}

Tras el pertinente proceso de evaluación según criterios de calidad editorial, en setiembre de 2005 CAICYT-CONICET ha clasificado a nuestra publicación con Categoría 1 (nivel superior de excelencia), con lo cual pasa a integrar el Catálogo Latindex (folio 14022). La Dirección de Revista veterinaria agradece a quienes colaboraron para obtener tan importante distinción. Ver: http://www.latindex. unam.mx/busquedas/catalogotitulo.html 